\title{
PHYSICAL VERSUS SOCIAL FEAR: A FUNDAMENTAL DICHOTOMY
}

\author{
Estelle Longin*, Mariam Chammat, Georges Chapouthier, Roland Jouvent
}

CNRS, CHU Pitié-Salpétrière, Paris, France

Received March 11, 2010; accepted March 25, 2010

\begin{abstract}
In this review we scrutinize the differences between two aspects of fear: physical fear and social fear. Based on the cognitive and behavioural differences in panic disorder and social phobia we try to correlate cerebral structures implicated in both kinds of fear. We study the neocortical control mechanisms over physical fear that render social fear more channelled. We also try to understand the dynamics and evolutionary origins of each, knowing that physical fear is more immediate while social fear has more social and evolved characteristics.
\end{abstract}

Key words: Emotion; Fear; Social Cognition; Behaviour

\section{INTRODUCTION}

Facing dangers presented in the environment animals less elevated in the phylogenetic tree are directly alerted by a pain sensibility called "nociception". They respond to it by withdrawal or by movements like retreating a part of the body or simply by flight. For the most elevated animals, here mainly vertebrates, dangers presented in the environment are processed in a differed manner. Even though low level immediate responses are possible they usually "evaluate" the consequences of their actions. This evaluation can be achieved though contributions of negative emotions that we can articulate in three fundamental concepts: stress, fear, and anxiety.

Stress as defined by Hans Selye is not fundamentally considered an emotion even though our modern usage of the word seems to consider it so. It usually refers to a "stressed person" to designate a person consciously going trough a rough period of time. Stress is rather seen as a global hormonal response of an organism that is put in harmful or unpleasant conditions. The present study is focused on more cerebral aspects of negative emotion. Darwin (1872) has already suggested the functional utility of fear; indeed, it represents the cerebral alarm elicited by a threatening stimulus. In vertebrates, fear and its mechanisms are supported by the limbic system. When the stimulus is not really present, when fear is temporally "differed", we can talk about anxiety which is rather a state of negative emotion that puts the limbic system in alert. When the stress generating stimulus is absent there can exist two types of anxieties: "legitimate" ones and "excessive" ones namely highly exceeding the "normal" alert reaction of the concerned organism. Whether legitimate (normal) or no justified (pathologic), anxiety can be assimilated to the cerebral response of stress. Whereas stress is a physiological response of the entire body, anxiety would be, in a way, its mental consequence.

Studies in animals point out clearly the idea of different anxiety levels from normal to pathologic conditions. Administration of anxiogenic or anxiolytic agents to rodents allow to define a "moderate" anxiety, useful for the well-functioning of the organism, and an "excessive" one (Clément \& Chapouthier, 1998). Behavioural responses to fear and anxiety can vary widely among situations, species, and even intraspecies. They can translate into an immobilisation, a "falling back" of the animal on itself ("freezing") or, at the contrary, into an active motor response like flight or fight. In many cases, in the mouse for example, the aggressive tendency is correlated to anxiety (Guillot \& Chapouthier, 1996), but there are exceptions (Venault \& Chapouthier, 2007). These fear, anxiety, and stress mechanisms can be found in most animals, in all

*Correspondence to: Estelle Longin, email: estellelongin@gmail.com 
vertebrates in particular. We can join them under the concept of "physical fear". However, for animals living in societies, like big apes and humans, another kind of "fear" appears, centred on more specific anxiety and stress components. This "social fear" results of society living and his constraints, and the inconvenience it can causes to the individual.

Likewise for humans, in physical danger situations fear is induced by the perception of a danger that threatens survival (aggression, gun, fire...): the risk is real and is directly perceived. In a social context however, (public speaking, or in front of a camera...) fear comes from mental constructions. A threatening characteristic is therefore actively attributed to a stimulus. Fear can therefore be elicited by the simple anticipation of the event since it is its representation that is anxiogenic even in the absence of the triggering stimuli. Social fear is readily likened to anxiety since the triggering stimulus is not necessarily present: it is rather a modulation of a pre-existing fear. The difference between physical and social fear therefore seems to reside in the idea of potential threat. Either the danger is real and involves high risk levels -fear would be elicited- or the situation is ambiguous, the threat possible and in this case it is anxiety and its associated behavioural correlates that would be elicited.

In our report, we want to show how the cerebral systems used in physical fear management (and in the associated anxiety) have been adapted to the management and control of social fear. Social fear, for obvious reasons, can't lead to extreme behaviour, especially an aggressive one, since it could be harmful for the group. Hence, we would like to show that basic limbic mechanisms of "physical fear" have to be controlled, canalised, by neocortical structures. In the first section of this article, based on Gray and McNaughton's (2000) theory, we start by exposing panic disorder and social phobia as models of physical and social fear respectively. We then describe cognitive and behavioural differences between these two pathologies. Based on these arguments, we identify cerebral structures implicated in these two kinds of fear.

\section{PHYSICAL FEAR AND SOCIAL FEAR}

Gray and McNaughton's thesis on animals and their effects on humans

Gray and McNaughton's (2000) theory mainly stemming from animal behaviour proposes a precise distinction between fear and anxiety. Based on functional, behavioural, and pharmacological aspects it suggests a link between orientation and emotion when faced with danger. On the one hand, the cerebral system controlling fear would induce the animal to move away from the threatening source. On the other hand, when the risk is ambiguous and needs to be evaluated, anxiety would lead to a movement towards the source of danger. These proposals are supported by the use of pharmacological substances in rodent: substances effective against panic disorder reduce flight behaviour (Blanchard, Griebel, \& Blanchard, 2001; Griebel, Blanchard, Agnes, \& Blanchard, 1995 ; Griebel et al., 1995), whereas substances effective against general anxiety disorder reduce danger exploration and evaluation, and limit defensiveness by reactions of intimidation ("weapons" exposition: teethes, claws...) or by attack (Blanchard, Griebel, Henrie, \& Blanchard, 1997). The authors suggest that the behaviours observed when faces with a dangerous situation constitute two groups, each reflecting the activation of a distinct cerebral system: fear or anxiety. Results obtained also entail the existence of a specific pathology related to the fear network dysfunction in human: panic disorder.

Based on this theory, and on similar grounds McNaughton and Corr (2004) suggest a defence model in humans and animals. In this model, "distance from the threatening stimuli" is introduced as a determining dimension of the activation of the cerebral network of fear or anxiety. According to this model, perception of close danger would solicit fear and would activate avoidance behaviours. Conversely, if danger is far, it would trigger anxiety and would lead to approaching the source of threat. A behavioural study seems to support this idea showing that direct or close threat stimuli presentation (angry faces) facilitates behaviours of avoidance whereas presentation of stimuli suggesting potential threat (fearful faces) facilitates approach behaviour (Marsh, Ambady, \& Kleck, 2005).

Consequently, facing risky situations, behaviours engaged in, seem to tend toward an "optimal" distance for which threat is identified but can be easily avoided. This distance, called "defence distance" by the authors would be inherent to everyone. Anxious persons in particular, would perceive danger as closer than it is in reality. Also, anxiolytics would not act directly on behaviour, but by increasing the perceived distance.

Nevertheless, a particular point specific to anxiety that is not taken into account in this model is threat anticipation. Indeed, we know that anxiety is not only activated in response to a distant stimulus but also when the danger is anticipated. In this last case, the reaction adopted will depend on the anxiety level that the person presents. The more anxious the individual is, the stronger his tendency to avoid a confrontation with the object of fear. In phobias, which are extreme and pathological forms, anxiety maintains threat at distance as a result of avoidance of dreaded situations. It seems that the distinction between approach and anxiety vs. fear and avoidance suggested by McNaughton and Corr is slightly more complex.

Fear and anxiety reactions can also be differentiated on a different basis. In the case of fear, reactions are more simple and intense like disorganised flight, and 
paralysing immobility. With anxiety, adopted behaviours are more complex and less intense: they are directed and organised in a goal when the threat is far away, and in this case they can lead to approaching the danger, or on the contrary, they can be linked to avoidance in order to maintain the danger at distance.

Beyond the notions of distance and orientation suggested above, intensity of threat also seems to be crucial. Indeed, activation of fear or anxiety also depends on the importance of the danger: anxiety is raised by small fears that take a disproportionate value, whereas panic and extreme behaviours are induced by big fears. This distinction mainly shows that neither the trait nor the state of anxiety can discriminate between panickers from non panickers and that they can't predict the severity of panic (Eser, Wenninger, Baghai, Schüle, \& Rupprecht, 2008). Individuals with high-trait anxiety would respond less intensely to the threat than individuals with low-trait anxiety (Perkins \& Corr, 2006).

It seems that a subtype of panic disorder (the one in which panic attacks are released without triggering stimulus and that is not accompanied by agoraphobia) could be considered a pathological model of the cerebral network of fear, referred to as "physical fear". In the following report, the term panic disorder referred to this special type of the pathology. Conversely, we know a pathological model of social fear: social phobia. Analysing the cognitive and behavioural differences between these two characteristic pathologies could elucidate the distinctive cerebral areas particular to each.

\section{Cognitive differences between social phobia and panic disorder}

Clark (1989) has proposed a cognitive view of the differences among anxious disorders. In his cognitivebehavioural theory Clark supposes that subjects with generalized anxiety disorder and panic disorder present different "cognitions". This hypothesis has been examined in the study of Breitholtz et al. (1999), based on an auto-observation method. For a period of two weeks, subjects report in a note-book the feelings, thoughts, images, and anxiety levels they experience during anxious events. Results underline a cognitive specificity: subjects with panic disorder report in a significantly more anxious events linked to physical dangers or disasters (accidents, death, for example), whereas subjects with generalized anxiety disorder express more worries about minor details or those linked to the interpersonal domain. In social phobia, which is subject of interest here, worries are clearly and specifically centred on social situations that can vary from a patient to another.

The cognitive divergences between panic disorder and social phobia could be linked, in part, to processing differences of physical threat situations and social threat situations. Indeed, the study of Rapee (1997) shows that, in a risky situation, the level of fear is more related to probability of the danger's occurrence. On the other hand, in a social situation the degree of fear depends on the control the subject has on the situation. Then, according to the situation the processing would differ, and this differentiation would be reflected by judgment biases. In panic disorder, a defective processing of physical danger situations would be linked to an overestimation of danger probability, provoking an excessive fear and very intense behaviours. In social phobia, the processing of social threat situations would be affected, the increase of patient's anxiety level would come from an underestimation of their capacity to control the situation.

The cognitive model of Beck and Emery (1985) also suggests a judgment bias in anxious subjects that leads them to underestimate their capacity of control. Cloitre et al. (1992) show a more precise distinction between panic disorder and social phobia according to the source of the threat, external or internal to the individual. This team used the "locus of control" scale of Levenson (1973) in patients with social phobia and panic disorder. This tool evaluates the proper perception of an individual of his capacity of control on events. Results corroborate a defect of processing specific to social situations or physical situations related to a locus of control, characteristic of the disorder. Compared to normal subjects, both clinical groups show reduced perception of control capacity but the origins are opposed in these two pathologies. Individuals with panic disorder explain their lack of control by the fact that events occur in an uncertain and non-controllable manner. In other terms, their lack of control is external; it comes from the threatening stimulus. Social phobics on the other hand view themselves as less competent than others; their lack of control comes from their wrong estimation of their own efficacy (inside orientation).

According to Chorpita and Barlow (1998) however, the lack of control would play a particularly important role in the development of anxiety. We can think that, in the case of panic disorder, the disorder would originate from reduced external control perception or in other terms from a perceived incapacity to manage the unexpected arrival of external events. Social phobia in return could be developed from a perceived incapacity to manage some kinds of situations. The specificity of each of the pathologies, physical or social, could come from an over-evaluation of risks: either the perceived physical danger or the threat induced by the presence of someone else. The lack of perceived control and danger over-evaluation are two components that would be translated by different processing biases for these two kinds of situations and fears. 


\section{Behavioural differences between physical and social fear}

The predisposition of an individual to confront to danger or to maintain him/herself at distance will determine the behaviour adopted in a situation of potential threat. We argue that subjects with panic disorder are characterised by an external locus of control whereas, in social phobics, the locus of control is internal. In a study (Miller \& Mulligan, 2002) on " risk taking » a correlation has been established between locus of control and the risk taking tendency. Subjects with an internal locus of control, such as social phobia, thinking that what happens depends on them, are less predisposed to risk taking. Subjects with an external locus of control, who think that what happens is arbitrary or that it depends on others, are more incline to the risk taking. The latter will therefore present less avoidance than people with an internal locus of control. Besides, facing a threatening stimulus or a dangerous situation, the nature of behaviours released by physical or social threat is easily distinguishable. Whereas physical threat activates simple and reflexive reactions, social threat elicits fine and flexible reactions in order to adapt the organism to more complex situations.

Panic attacks are physiological manifestations of fear that arise very fast. They are characterised by a paroxysm that is reached very rapidly (seconds or minutes), and by a strong impression of loss of control. They can be translated by an immediate flight or, on the contrary, by a marked motor inhibition that could lead to a total staged state comparable to freezing in animals. Among psychical symptoms we can find depersonalisation (not knowing who we are) or derealisation (modification of the environment perception). Panic attacks arise most of the time in phobic patients, when they are confronted to the object of their fear.

Primary symptoms of a panic attack show the automatism with which it is declared. Behaviours are very intense; they are induced in a fast and reflexive manner, and without conscious planification.

As we argued, anxiety mechanisms would take over when distance to threat is more important. In the case of a distant danger, responses such as exploration and risks evaluation are more moderate and more organised. They appeal to a regulation that attenuates extreme and intense behaviours we described. Therefore, the regulation of anxiety mechanisms transforms the disorganised flight into an oriented avoidance, whereas the immobilisation due to a marked motor inhibition becomes blurred and, in a way, more controlled. A first type of "freezing" coming from the overactivation of fear system, would be the translation of an emotional interference that deteriorates the activation or the progress of motor and cognitive processes. A second type would be, on the contrary, a state of increased vigilance conducted by anxiety.

The complexity of social interactions requires a much more significant behavioural regulation. Mechanisms implicated in fear expression, in particular, have to be modulated in a way to avoid behaviour that would hinder social cohesion. For social animals, like for humans, there appears to be a new type of defence strategy: submission. This kind of defence helps avoiding destructive confrontation between conspecifics. It has been proposed that, in humans, blushing or lowering the gaze, provoked in anxiogenic social situations, can be assimilated to persistent signals indicative of a submission in animals (Shuhama, DelBen, Loureiro, \& Graeff, 2007).

Behaviour that induced by a social fear is characterised by an inhibition that can be strongly marked. When fear is intense, the anxiogenic social elements such as visual contact or certain types of situations are avoided. Even if it is not striking at first, eye avoidance can be seen as a defence strategy because this behaviour circumvents any sort of confrontation. In Primates, for example, the duration of eye contact has a different meaning according to the species. For the vigilant ones, the gaze is perceived as a threat and will be avoided in most of social interactions (Emery, 2000). In Humans, the perception of a face and of its expressions is at the centre of social interaction. Eye contact is particularly important because it expresses attention and engagement. In social phobia, the gaze is perceived as a source of negative social evaluation, that's why it is often avoided.

Clinical observations of this behaviour have been verified by Horley et al. (2004) who measured the scanpaths of subjects viewing different facial expressions. Whereas in normal controls scanpath tends to form a triangle between the more salient characteristics of the face (the eyes and the mouth), social phobics present a hyperscanning: their eye movements scan all the face. This behaviour reflects a hypervigilance due to the high level of anxiety of the subjects for this type of stimuli. Moreover, number and time of visual fixations are strongly reduced in the eyes area. Social fear engages behaviour that is more moderate and subtle than physical fear, and probably implicates learning mechanisms. Adapting to social situations, overall when they are anxiogenic, requires good regulation capacities via inhibition, which are acquired in particular by experience.

\section{From facial expressions to context}

The study of danger perception in humans is usually done via the study of angry and that of fearful facial expressions. It remains that in situations of real danger, the context is a crucial element to determine and evalu- 
ate the level of danger to which one is exposed. Perception of fear expressions indicates a potential danger but, the localization and the determination of the risk requires additional information gathering from the environment. Angry expressions would rather point out a direct threat, since the person depicting that expression could be the source of danger. Nevertheless, presented alone, an angry expression remains ambiguous as it is not informative of the cause of anger neither of the significance and direction of threat.

Serotonin plays an important role in modulation of facial expression recognition which shows that angry faces would be less likely perceived as a direct threat. This cerebral neuromodulator plays an important role in the regulation of defensive behaviours. Various arguments such as the efficacy of serotonin reuptakers (low serotonin level in synapse) in some anxious disorders indicate that serotonin would facilitate anxiety related behaviour, as potential threat assessment, and that it would inhibit reactions related to panic, generated by proximal threat. On the one hand, since fearful faces are considered as a potential threat, serotonin would enhance these expressions' recognition. On the other hand, if angry expressions are perceived as a direct or proximal threat, detection of these expressions should be deteriorated. Taken together, studies (see Del-Ben, Ferreira, Alves-Neto, \& Graeff, 2008) tend to show that an increase of serotoninergic neurotransmission improves fear recognition without deteriorating anger recognition. In other words, an angry facial expression identified in a member of the same species would not necessarily signal a direct threat, or would be not sufficient to be perceived as such. Indeed in humans, apart from social phobia, the meaning attributed to a faces expressing anger depends strongly on the context. In a real situation, perception of a fear or anger facial expression is not sufficient to evaluate the level of danger. From experience, we know that other signals are necessary. These are gathered in part from the subject's attitude, his posture, body expressions, and the tone of his voice. Even though these cues have important impact on facial expression processing, very few studies have explored this effect. The recognition of an emotion transmitted by a face is influenced by the body expression or prosody, and systematically facilitated when the emotion is congruent (de Gelder et al., 2006 for review; Aviezer et al., 2008).

Beyond the bodily cues pointing to fear, environment and "physical" context, can also be very informative. It has been demonstrated that fearful scene presentation activates the amygdala (Hariri, Tessitore, Mattay, Fera, \& Weinberger, 2002). In this case context would influence the manner with which a face is encoded. The N170 component, a wave specifically sensitive to faces, presents larger amplitude when a fear face is presented in a fearful context, compared to a neutral context (Righart \& de Gelder, 2006).

In summary, we went from the Gray and McNaughton's (2000) and McNaughton \& Corr (2004) theories of defensive system in animals to make a distinction between physical fear and social fear in human. Taking social phobia and panic disorder as human pathological models of social and physical fears respectively, we reviewed cognitive and behavioural differences between these two pathologies. This distinction allowed us to define physical fear as a specific activation of the fear cerebral system. Orientated on external danger physical fear goes off by proximal threat detection, triggering rapid and intense behaviours. On the other hand, social fear would rely on more distant or anticipatory danger making this kind of fear more relevant of the anxiety cerebral system. Indeed, social phobia is accompanied by more internal worries and more controlled behavioural responses such as inhibition in particular. Moreover, having established this difference will allow us to look for distinct cerebral supports.

\section{THE ANATOMICAL SUPPORTS QUEST From cognitive arguments}

A comparison of physical and social fear tends to show different neural correlates for each. First of all, the cognitive demarcation between panic disorder and social phobia suggests that, in panic disorder, where worries are centred on integrity and organism survival, low-level mechanisms supported by limbic structures of the neural network of fear could be affected. Indeed, physical situation processing seems to be biased. Yet, probability and physical danger level evaluations seem to be rather "primitive". They produce basic survival behaviour and constitute mechanisms shared by a large number of animal phyla, in particular vertebrates.

In social phobia on the other hand, different arguments suggest that neocortical structures are involved. For example, processing and representation of social situations are high-level processes that require engagement of more elevated cerebral structures. Alteration of social situation representation in social phobics could come from a defect in these structures.

Moreover, the notion of "self" -meaning all that makes reference to the self- (Fossati et al, 2003) seems important, and its substratum could be hit in social phobia. Indeed, the principal worry of patients is being helpless or mismanaging social situations. A wrong representation of the self, built from a wrong estimation of the subject's proper skills, could be at stake. An fMRI study (Moran, Macrae, Heatherton, Wyland, \& Kelley, 2006) shows specifically that the emotional and cognitive components of self are managed by neocortical areas: the medial prefrontal cortex and the cingular ventral anterior cortex, respectively. 
Another "cognitive" argument that supports the implication of these cortical regions of the self or ego is that of the switch from first person to second person. In a social interaction, social phobics spontaneously position themselves as being observed ("1st person") and judged by the others. It is hard for them to see themselves as the observer. This position implies a transfer to a new point of view, coming from oneself but in the same time detached, somewhat resembling a "2nd person". By consequence, this switch from an egocentric perspective to an allocentric perspective seems to be affected in social phobia. This transition would depend of certain flexibility which has been put forward experimentally in studies on episodic memory and the change of point of view (Libby \& Eibach, 2002; Libby, Eibach, \& Gilovich, 2005). Such abstract cognitive considerations should also appeal to neocortical structures. Moreover, they require introspection and mental state attribution processes, which are strongly affected in social phobics and which rely on ventro-medial prefrontal cortex (Jenkins, Macrae, \& Mitchell, 2008).

\section{From behavioural descriptions}

The fast and reflexive reactions triggered by panic attacks indicate that low-level structures, subcortical, are implied. Many studies include the periaqueductal gray in the neurology of panic disorder but, this structure of midbrain would not be the only one implicated. An increase of amount of gray matter of the insula and the superior temporal cortex has been noted as well as a reduction of amount of gray matter of the cingular anterior cortex while hippocampic, parahippocampic areas seem to be subject to functional abnormalities (Del-Ben \& Graeff, 2009).

A recent neuroimaging study (Mobbs et al., 2007) tested the hypothesis according to which cerebral activity associated to distal threat detection would provoke an activity in regions associated to complex decision making, such as ventro-medial prefrontal cortex and anterior cingular cortex. On the other hand, detection of proximal threat would engage mesencephalic structures, including the periaqueductal gray. In rodents, this region is implicated in reflexive behaviours like flight and freezing (Schenberg et al., 2005).

In this experiment, the cerebral activity of volunteers is recorded in fMRI during a pursuit by a predator in a virtual labyrinth. The predator is also capable of inflicting strong or light intensity pain delivered in the form of an electrical impulse on the hand. Results obtained confirm the pre-established hypothesis: signals show that, when the predator approaches, cerebral activity moves from the ventro-medial prefrontal cortex to the periaqueductal gray.

Periaqueductal gray's activity measured in this task reflects the increase of subject's panic. Its activity is correlated to the dread of being caught by the predator and a decrease in confidence to escape. Moreover, it has been observed in humans that electrical stimulation of this region activates the emotional sensations of fear (see Sewards \& Sewards, 2003). These results support the implication of periaqueductal gray in the pathophysiology of panic disorder.

It is also interesting to note that defensive behaviour induced in order to survive is managed by this circuitry which is closely related to nociception. Indeed, the periaqueductal gray is implicated in the pain circuit and, in Mobbs et al.'s study, its maximal expression is obtained when the subject is waiting for an intense pain. Taken together these data indicate that the periaqueductal gray is at the centre of the fear cerebral network and that it could be crucial in panic disorder. Also implicated in the pain circuitry, the periaqueductal gray participates to trigger rapid and reflexive survival behaviour in order to deal with an emergency life threatening situation.

Ventro-medial prefrontal cortex activity is converse to the periaqueductal gray activity: it increases when danger moves away. In other words, this structure would be rather implicated in the neural circuitry of anxiety. According to the authors, this prefrontal activity represents processes comparing possible behaviours in order to choose the most efficient strategy to avoid the threat. This hypothesis supports Damasio et al.'s (1991) somatic markers' hypothesis, according to which patients with ventro-medial prefrontal cortex lesions would be unable to activate somatic signals required for strategy selection. In normal subjects, ventro-medial prefrontal cortex would activate these markers in order to reproduce emotional sensations associated to an anxious state.

Mobbs et al.'s (2007) data show that, two seconds before the predator encounter, when the subject is waiting for a threat, an increase of activity is recorded in the anterior rostral and ventral cingular cortex, the medial orbito-frontal cortex and the ventro-medial prefrontal cortex. The authors suggest that, when the danger is detected, the increased activity recorded in the rostral anterior cingular cortex is associated to a response conflict between "staying" and "flying". The increased activity in the medial orbito-frontal cortex would represent the value of the threat, its intensity.

Therefore, as suggested, physical fear would mainly rely on a subcortical structure, the periaqueductal gray. When danger moves away, the anxiety network takes over and recruits neocortical regions to modulate behavioural responses and adopt the most strategic choice.

In social fear, the inhibition mechanisms and the gaze avoidance that characterize it support a large implication of neocortex too, in particular of the frontal lobe (Aron, Robbins, \& Poldrack, 2004). 
A commonly used paradigm that helps study and measure behavioural response inhibition is the Go / NoGo task. This task is designed in a way to increase response tendency. Indeed trials that induce responses are more frequent than trials that don't.

A recent fMRI study showed that the posterior inferior frontal gyrus was specifically activated for response inhibition (Chikazoe et al., 2009). Since social anxiety is linked to a behavioural inhibition, this region could be involved. Moreover, the orbito-frontal cortex seems to play an important role: symptoms of fronto-temporal dementia (Viskontas, Possin, \& Miller, 2007; Peters et al., 2006) and lesional studies of this region (Starkstein \& Robinson, 1997) show that it is closely related to behavioural inhibition, specifically in social contexts.

On the other hand, gaze avoidance can be compared to a well-studied movement in oculomotricity: the "antisaccade". This ocular movement consists of directing gaze in the opposite side of a visual stimulus. As gaze avoidance, this movement requires an attentional disengagement of the stimulus to move to the opposite side. The antisaccade task is frequently used to test two abilities: first the suppression of a reflexive prosaccade, a movement directed toward the visual stimulus, and then the generation of a voluntary motor command, a movement directed to the other side. This task gained importance after a study on frontal lesion patients (Guitton, Buchtel, \& Douglas, 1985). Indeed, these subjects produce more errors than controls. They can't execute the task, they constantly direct the eyes toward the visual stimulus. They are not able to correct their movements and move their gaze in the opposite side. Therefore, an increasing number of studies gave interest to the antisaccade task considering the augmentation of the error rate as a measure of the frontal lobe function. Lesion and fMRI studies that used this paradigm highlight the recruited regions (PierrotDeseilligny, Müri, Nyffeler, \& Milea, 2005). They show that the dorso-lateral prefrontal cortex is solicited for a reflexive saccade inhibition, to correct the saccade directed toward the stimulus. The frontal eye field would be the principal region recruited to start an antisaccade intentionally. We can thus conceive that the intervention of these two regions would be important in gaze avoidance observed in social phobia.

Taken together these data confirm the predominant implication of neocortex and in particular of prefrontal cortical regions when fear is social.

\section{A neural circuitry modulated by serotonin?}

These two regions put forward by Mobbs et al. (2007) experience, seem to be complementary. As the ventromedial prefrontal cortex gives favour to the planification and decision making to adopt the most strategic behaviour, the periaqueductal gray engages rapidity and automatic behaviour. Mobbs et al. (2007) suggest that defensive behaviour would be determined by a balance of activity between regions implicated in the prosencephalon (cortex) and the mesencephalon (periaqueductal gray).

As we discuss earlier, serotonin is anxiogenic in the anterior brain, and decreases panic in the periaqueductal gray (Graeff, 2004). Serotonin seems to be a good candidate to regulate this balance of activity. We can now consider a system of defence relying on a large neural network where serotoninergic regulation could adapt behavioural reactions according to danger imminence. When danger is ambiguous or anticipated, anxiety and risk evaluation would be activated. When threat gets closer, behaviour related to fear would be activated and would become more intense with proximal distance to danger. An increase of serotonin in this system would facilitate behaviour related to anxiety and would inhibit behaviour implicated in fear reactions, and vice versa. Moreover, serotonin sensibility could also be involved in social fear, because social phobic patients would present an increased sensibility of post-synaptic 5-HT 1A and 5-HT 2 receptors (Aouizerate, Martin-Guehl, \& Tignol, 2004). Prefrontal cortex would be more solicited by anxiety in order to establish different options of risk evaluation and escape strategies. When danger is close, automated and rapid strategies are opted for. Subcortical structures of fear are therefore activated to trigger automatic survival behaviour. A deterioration of regulation in this network could lead non-adapted behaviour, such as excessive anxiety related to minor risk evaluation.

In summary, we used cognitive and behavioural arguments to highlight cerebral differences between physical and social fear. On the one hand, we argued that physical fear relies on subcortical structures of the limbic system with the periaqueductal gray at the centre of this network. On the other hand, social fear appears to be a more canalised fear because it is under the control of prefrontal cortical structures such as the ventro-medial prefrontal cortex. Finally, we supported the idea that defensive behaviour could be sustained by a single neural circuitry modulated by serotonin. This neuromodulator would regulate the balance of activity between cortical and subcortical structures implicated in physical and social fear systems.

\section{CONCLUSION}

Taken together the different works presented here reveal a fundamental dichotomy in anxious behaviour processing: one that would oppose physical fear and social anxiety.

Physical fear, strongly rooted in the animal reign, would produce intense and rapid behaviour of flight or immobility, in response to a directly threatening stimulus. It would be managed by subcortical mechanisms among which the periaqueductal gray, also 
implied in primary expression of nociception, would play a fundamental role. Panic disorder in humans is clearly related to this physical fear, common to most animals. Social fear on the other hand, like social phobia in humans, would call for more deferred behaviour and would participate to what we can call a generalized anxiety. It would translate difficulties of optimal strategy analysis, by a defect in social stimuli control such as gaze, facial expression or environmental context. On the anatomical plan, it would rely on neocortical processes, particularly prefrontal ones. Finally, serotoninergic mechanisms would play an important role for the switch from a panic fear more immediate and a social anxiety more mediate. These mechanisms would facilitate more controlled and channelled behaviour related to anxiety and would inhibit more immediate and subcortical behaviour related to physical fear.

This dichotomy between immediate behaviour, profoundly rooted in animality, and more deferred behaviour, more specific of evolved animals and particularly social animals, is found in many phenomena of the living. The switch from painful nociception to suffering, the transformation of alert communication in complex systems, like birds singing or human language, or the switch from an awareness of environment to a reflexive consciousness (conscious to be conscious) are some examples among others of deferred or mediated experiences, that emerge in most evaluated animals and that find their maximal extension in human beings. An analysis of fear and of its anatomical mechanisms, as we did, offers us an excellent example.

\section{REFERENCES}

Aouizerate, B., Martin-Guehl, C., \& Tignol, J. (2004) . Neurobiology and pharmacotherapy of social phobia. Encéphale, 30 (4), 301-313.

Aron, A. R., Robbins, T. W., \& Poldrack, R. A. (2004). Inhibition and the right inferior frontal cortex. Trends in Cognitive Sciences, 8 (4), 170-177.

Aviezer, H., Hassin, R. R., Ryan, J., Grady, C., Susskind, J., Anderson, A., et al. (2008). Angry, disgusted, or afraid? Studies on the malleability of emotion perception. Psychological Science, 19 (7), 724-732.

Beck, A. T., \& Emery, G. (1985). Anxiety disorders and phobias: A cognitive perspective. New York: Basic books.

Blanchard, D. C., Griebel, G., \& Blanchard, R. J. (2001). Mouse defensive behaviors: pharmacological and behavioral assays for anxiety and panic. Neuroscience and Biobehavioral Reviews, 25 (3), 205-218.

Blanchard, R. J., Griebel, G., Henrie, J. A., \& Blanchard, D. C. (1997). Differentiation of anxiolytic and panicolytic drugs effects on rat and mouse defense test batteries. Neuroscience and Biobehavioral Reviews, 21 (6), 783-789.
Breitholtz, E., Johansson, B., \& Öst, L. G. (1999). Cognitions in generalized anxiety disorder and panic disorder patients. A prospective approach. Behaviour Research and Therapy, 37, 533-544.

Chikazoe, J., Jimura, K., Asari, T., Yamashita, K., Morimoto, H., Hirose, S., et al. (2009). Functional dissociation in right inferior frontal cortex during performance of go / no-go task. Cerebral Cortex, 19 (1), 146-152.

Chorpita, B. F., \& Barlow, D. H. (1998). The development of anxiety: the role of control in the early environment. Psychological Bulletin, 124 (1), 3-21.

Clark, D. M. (1989). Anxiety states: Panic and generalized disorder. In K. Hawton, P. M. Salkovskis, J. Kirk \& D. M. Clark (Eds.), Cognitive-behaviour therapy for psychiatric problems: A practical guide (pp. 52-96). Oxford, United Kingdom: Oxford University Press.

Clément, Y., \& Chapouthier, G. (1998). Biological bases of anxiety. Neuroscience Biobehavioral Reviews, 22 (5), 623633.

Cloitre, M., Heimberg, R. G., Liebowitz, M., \& Gitow, A. (1992). Perceptions of control in panic disorder and social phobia. Cognitive Therapy and Research, 16 (5), 569-577.

Damasio, A. R., Tranel, D., \& Damasio, H. (1991). Somatic markers and the guidance of behaviour: theory and preliminary testing. In Levin, H. S., Eisenberg, H. M., Benton, A. L. (Eds.), Frontal Lobe Function and Dysfunction (pp. 217-229). New York: Oxford University Press.

Darwin, C. R. (1872). The expression of the emotions in man and animals. John Murray, 1st ed., London.

de Gelder, B., Meeren, K. M., Righart, R., van den Stock, J., van de Riet, W. A. C., \& Tamietto, M. (2006). Beyond the face: exploring rapid influences of context on face processing. Progress in Brain Research, 155, 37-48.

Del-Ben, C. M., Ferreira, C. A. Q, Alves-Neto, W. C., \& Graeff, F. G. (2008). Serotonergic modulation of face-emotion recognition. Brazilian Journal of Medical and Biological Research, 41, 263-269.

Del-Ben, C. M., \& Graeff, F. G. (2009). Panic disorder: is the PAG involved? Neural Plasticity, 2009, 108-135.

Emery, N. J. (2000). The eyes have it: the neuroethology, function and evolution of social gaze. Neuroscience and Biobehavioral Reviews, 24, 581-604.

Eser, D., Wenninger, S., Baghai, T., Schüle, C., \& Rupprecht, R. (2008). Impact of state and trait anxiety on the panic response to CCK-4. Journal of Neural Transmission, 115 (6), 917-920.

Fossati, P., Hevenor, S. J., Grady, C., Keightley, M. L., Craik, F., \& Mayberg, H. (2003). In search of the emotional self: an FMRI study using positive and negative emotional words. American Journal Psychiatry, 160 (11), 1938-1945.

Graeff, F. G. (2004). Serotonin, the periaqueductal gray and panic disorder. Neuroscience and Biobehavioral Reviews, 28, 239-259.

Gray, J. A. \& McNaughton, N. (2000). The Neuropsychology of anxiety: an enquiry into the functions of the septohippocampal system (2nd ed.). Oxford, United Kingdom: Oxford University Press. 
Griebel, G., Blanchard, D. C., Agnes, S., Blanchard, R. J. (1995). Differential modulation of antipredator defensive behavior in Swiss-Webster mice following acute or chronic administration of imipramine and fluoxetine. Psychopharmacology, 120 (1), 57-66.

Griebel, G., Blanchard, D. C., Jung, A., Lee, J. C., Masuda, C. K., \& Blanchard, R. J. (1995). Further evidence that the mouse defense test battery is useful for screening anxiolytic and panicolytic drugs: effects of acute and chronic treatment with alprazolam. Neuropharmacology, 34 (12), 1625-1633.

Guillot, P. V., \& Chapouthier, G. (1996). Inter-male aggression and dark/light preference in ten inbred mouse strains. Behavioral Brain Research, 77, 211-213.

Guitton, D., Buchtel, H. A., \& Douglas, R. M. (1985). Frontal lobe lesions in man cause difficulties in suppressing reflexive glances and in generating goal-directed saccades. Experimental Brain Research, 58, 455-472.

Hariri, A. R., Tessitore, A., Mattay, V. S., Fera, F., \& Weinberger, D. R. (2002). The amygdala response to emotional stimuli: a comparison of faces and scenes. Neuroimage, 17, 317-323.

Horley, K., Williams, L. M., Gonsalvez, C., \& Gordon, E. (2004). Face to face: visual scanpath evidence for abnormal processing of facial expressions in social phobia. Psychiatry Research, 127, 43-53.

Jenkins, A. C., Macrae, C. N., \& Mitchell, J. P. (2008). Repetition suppression of ventromedial prefrontal activity during judgments of self and others. PNAS, 105 (11), 4507-4512.

Levenson, H. (1973). Multidimensional locus of control in psychiatric patients. Journal of Consulting and Clinical Psychology, 41 (3), 397-404.

Libby, L. K., \& Eibach, R. P. (2002). Looking back in time: self-concept change affects visual perspective in autobiographical memory. Journal of Personality and Social Psychology, 82 (2), 167-179.

Libby, L. K., Eibach, R. P., \& Gilovich, T. (2005). Here's looking at me: the effect of memory perspective on assessments of personal change. Journal of Personality and Social Psychology, 88 (1), 50-62.

Marsh, A. A., Ambady, N., \& Kleck, R. E. (2005). The effects of fear and anger facial expressions on approach- and avoidance-related behaviours. Emotion, 5 (1), 119-124.

McNaughton, N., \& Corr, P. J. (2004). A two-dimensional neuropsychology of defence: fear/anxiety and defensive distance. Neuroscience and Biobehavioral Reviews, 28, 285-305. Miller, R. L., \& Mulligan, R. D. (2002). Terror management: The effects of mortality salience and locus of control on risktaking behaviors. Personality and Individual differences, 23 (7), 1203-1214.

Mobbs, D., Petrovic, P., Marchant, J. L., Hassabis, D., Weiskopf, N., Seymour, B., et al. (2007). When fear is near: threat imminence elicits prefrontal-periaqueductal gray shifts humans. Science, 317 (5841), 1079-1083.

Moran, J. M., Macrae, C. N., Heatherton, T. F., Wyland, C. L., \& Kelley, W. M. (2006). Neuroanatomical evidence for distinct cognitive and affective components of self. Journal of Cognitive Neurosciences, 18 (9), 1586-1594.
Perkins, A. M., \& Corr, P. J. (2006). Reactions to threat and personality: psychometric differentiation of intensity and direction dimensions of human defensive behaviour. Behavioural Brain Research, 169, 21-28.

Peters, F., Perani, D., Herholz, V., Beuthien-Baumann, B., Sorbi, S., Pupi, A., et al. (2006). Orbitofrontal dysfunction related to both apathy and désinhibition in fronto-temporal dementia. Dementia and Geriatric Cognitive Disorders, 21 (56), 373-379.

Pierrot-Deseilligny, C., Müri, R. M., Nyffeler, T., \& Milea, D. (2005). The role of the human dorsolateral prefrontal cortex in ocular motor behaviour. Annals of the New York Academy of Sciences, 1039, 239-251.

Rapee, R. M. (1997). Perceived threat and perceived control as predictors of the degree of fear in physical and social situations. Journal of Anxiety Disorders, 11 (5), 455-461.

Righart, R., \& de Gelder, B. (2006). Context influences early perceptual analysis of faces - An electrophysiological study. Cerebral Cortex, 16 (9), 1249-1257.

Schenberg, L. C., Póvoa, R. M., Costa, A. L., Caldellas, A. V., Tufik, S., \& Bittencourt, A. S. (2005). Functional specializations within the tectum defence systems of the rat. Neuroscience Biobehavioral Reviews, 29 (8), 1279-1298.

Sewards, T. V., \& Sewards, M. A. (2003). Representations of motivational drives in mesial cortex, medial thalamus, hypothalamus and midbrain. Brain Research Bulletin, 61, 25-49.

Shuhama, R., Del-Ben, C. M., Loureiro, S. R., \& Graeff, F. G. (2007). Animal defense strategies and anxiety disorders. Anais da Academia Brasileira de Ciencas, 79 (1), 97-109.

Starkstein, S. E., \& Robinson, R. G. (1997). Mechanism of desinhibition after brain lesions. Journal of Nervous and Mental Disease, 185 (2), 108-114.

Venault, P., \& Chapouthier, G. (2007). From the Behavioral Pharmacology of Beta-Carbolines to Seizures, Anxiety, and Memory. The Scientific World Journal, 7, 204-223.

Viskontas, I. V., Possin, K. L., \& Miller, B. L. (2007). Symptoms of fronto-temporal dementia provide insights into orbitofrontal cortex function and social behaviour. Annals of the New York Academy of Sciences, 1121, 528-545. 